\title{
Interventional Indirect Effects: A Novel Approach to Optimise Complex Psychological Therapies to Treat Depression for Scale-up in Goa, India
}

Nadine Seward ( $\sim$ Nadine.seward@kcl.ac.uk)

Kings College London https://orcid.org/0000-0002-4821-9437

Stijn Vansteelandt

University of Ghent

Darío Moreno-Agostino

UCL

Vikram Patel

Harvard

Ricardo Araya

King's College London

\section{Research}

Keywords: Causal mediation analysis, Interventional (in)direct effects, Behavioural Activation for Depression, Psychological therapies Task-sharing

Posted Date: October 29th, 2021

DOI: https://doi.org/10.21203/rs.3.rs-977875/v1

License: (c) (i) This work is licensed under a Creative Commons Attribution 4.0 International License. Read Full License 


\section{Abstract}

Introduction: Understanding how and under what circumstances complex psychological therapies work (or not) is important to bring evidence-informed intervention to scale, especially in resource poor settings. However, current methods do not apply methodology that account for the underlying complexity of these interventions including the interplay between implementation outcomes, implementation strategies and mechanisms. Here we apply a robust mediation analysis to address these issues to data from the Healthy Activity Program (HAP) trial -a psychological intervention for depression delivered using taskshifting with lay counsellors in Goa India.

Methods: Interventional in(direct) effects were used to simultaneously decompose the total effect of the intervention on depression symptoms measured through the Patient Health Questionnaire (PHQ-9). The following indirect effects were considered: fidelity of receipt including number of sessions and homework completed; behavioural activation according to an adapted version of the Behavioural Activation for Depression Short Form (BADS-SF), and extra sessions offered to participants who did not respond to the intervention.

Results: Of the total effect of the intervention measured through the difference in PHQ-9 scores between treatment arms (mean difference: $-2.2,95 \%$ bias-corrected $\mathrm{Cl}$ : $-3.2,-0.8$ ), 45\% was mediated through improved levels of behavioural activation $(-1.0,-1.3,-0.6)$. There was little evidence to support the mediating role of characteristics of the sessions nor the extra sessions offered to participants who did not respond to the treatment.

\section{Conclusions}

Findings from our analyses have demonstrated how interventional (in)direct effects can be applied to understand how implementation research programmes can be optimised for scale-up. Our results highlight the importance of sessions focusing on behavioural activation to improve symptoms of depression. Targeting non-responders with strategies other than extra therapy sessions has the potential to improve depression outcomes at a population level.

\section{Contributions To The Literature}

- Several trials have demonstrated the effectiveness of task-sharing to deliver psychological therapies; however, there is still a large treatment gap in bringing this evidence-informed care to scale.

- We used interventional (in)direct effects to simultaneously account for mechanisms of action and implementation outcomes to address the complexity of these programmes.

- Targeting levels of behavioural activation can help to improve recovery from depression, however the fidelity by which the intervention was received, did not mediate an improvement in depression.

- To optimise HAP for scale-up, more is needed to adapt the intervention to target non-responders other than offering up to two extra sessions. 


\section{Introduction}

Depression is a common mental health disorder that affects an estimated 300 million people worldwide and is the leading mental health cause to the global burden of disease.(1) Depression is also associated with excess mortality and morbidity as well as profound social and economic consequences.(1) Despite this, only $10 \%$ or fewer people with depression in low- and middle-income countries (LMICs) have access to effective treatment.(1)

Psychological treatments are the recommended first-line intervention for depression according to the WHO's Mental Health Gap Action Programme (mhGAP) as they have been shown to be just as effective as pharmacological interventions and have a sustained effect over time.(2) However, important barriers to accessing these treatments in LMICs exist, in particular the lack of trained professionals.(3) This has led to the design of briefer treatments and task-sharing the interventions to trained non-specialists.(4-7) Trials using different therapeutic approaches for depression such as psychoeducation, problem solving, and behavioural activation, through a task-sharing modality, have demonstrated varying levels of effectiveness in LMICs. (8-10)

Behavioural activation for depression is particularly applicable to LMICs as lay health workers with limited training can effectively deliver the intervention.(11) Behavioural activation improves symptoms of depression mainly by the mechanism of increasing the number of enjoyable or meaningful activities. There is good but not undisputed evidence to support the effectiveness of behavioural activation for depression in LMICs $(8,12-14)$.

Reasons to explain these differences in effectiveness from different trials may be related to the contexts where the interventions are delivered (middle vs low-income country or urban vs rural), the conditions studied (perinatal depression or comorbid hypertension and depression), mode of delivery (mHealth vs task sharing) and other underlying factors that are often not accounted for.

Characteristics of the sessions are known to influence depression outcomes through different pathways. As an example, evidence suggests that the number of sessions is an important predictor of recovery, with five to six sessions required as a minimum to improve depression outcomes.(12,15) Analyses that account for the multiple and often interacting pathways through which an intervention operates including both characteristics of the sessions and mechanisms such as behavioural activation will help to determine how to optimise different components in preparation for future scale-up. Comparing findings across different studies will provide insight into what works for whom and under what circumstances.

The Healthy Activity Programme (HAP)

One of the most successful trials of a lay counsellor-delivered psychological intervention for depression from a LMIC is the HAP trial. (12) HAP is an adapted form of behavioural activation delivered by lay counsellors on a face-to-face modality for participants with moderate to severe depression in primary care settings in Goa, India. Findings suggest that HAP improved remission from depression both at the 
end of the trial and at 12 months follow-up. However, little is known surrounding how HAP improved recovery from depression, other than findings from a mediation analysis that found $58 \%$ of the total effect was mediated through improved activation measured using the BAD-SF.(16)

Given the potential to bring this intervention to scale, it is important to understand how the HAP intervention can be optimised. The interventional (in)direct effects, is the latest in a series of recent advancements to causal mediation analyses that allows for the decomposition of the total effect of a complex intervention into multiple indirect effects that can characterise specific mechanisms and characteristics of the sessions.(17)

We therefore estimated interventional (in)direct effects based on data collected both at three and 12 months after the trial started for the following mediators: characteristics of the sessions (i.e. number of sessions and proportion of homework completed), behavioural activation, and extra sessions a participant received if they did not respond to treatment. It is hoped that findings will provide further insights into what components of the intervention worked (or not).

\section{Methods}

We used data from a previously conducted randomised controlled trial (HAP) that took place between October 2013 and July 2015 in primary health care centres in Goa, India, that included information on symptoms of depression and important mediators at three and 12 months after the trial started. Details of the original trial can be found in previous publications. $(12,16)$ The original trial was registered with the ISRCTN registry, number ISRCTN95149997.

\section{Design}

HAP was a parallel arm, individually randomised controlled trial with equal allocation of participants between arms. Participants aged between 18-65 years were recruited from 10 primary health centres. Eligibility criteria included a probable diagnosis of moderately to severe depression determined with a PHQ-9 score greater than 14. Pregnant women and participants who needed urgent medical attention or who were unable to communicate were excluded.

\section{The intervention}

The intervention (HAP) was a manualised psychological intervention based on behavioural activation for depression that primarily involved strategies to increase the number of enjoyable activities a person engaged with. $(12,18)$ Other strategies were also included after exploring their acceptability, appropriateness and feasibility in the local context including need-based strategies that addressed interpersonal triggers, problem-solving, relaxation and enlisting social support tailored to the specific need of the individuals.(19)

The experimental arm received up to eight sessions (described below) that lasted between 30 and 40 minutes at weekly intervals over a 3-month period. The sessions were usually face to face at the Primary 
Health Centre, or the patient's home. Telephone sessions were used only when strictly necessary. The intervention was organized in three phases. The first phase (sessions 1-2) was primarily used to engage the participant, establish an effective relationship, explain the objectives of the sessions including behavioural activation, and to elicit a commitment for the HAP intervention. The middle phase (sessions 3-4) assessed activation targets and encouraged activation, identifying barriers to activation, and learning to overcome these and how to solve or cope with life problems. The ending phase (sessions 5-6) reviewed and strengthened gains the patient made during treatment in order to prevent relapse. If a participant did not respond to treatment by the third or fourth session, two additional middle-phase sessions were offered resulting in these patients attending a total of seven to eight sessions.

HAP was delivered by lay counsellors who had completed at least the 10th grade of education and were fluent in local languages. Counsellors were also required to meet pre-defined competency standards.(20) Training took place over a three-week period. Counsellors received weekly peer-led supervision in groups and individual supervision twice a month.

Enhanced usual care (EUC) was offered to participants in both arms of the trial.(12) EUC involved screening results for depression being shared with both patients and physician. Physicians were also trained on how to use a contextualised version of the mhGAP guidelines, including when and where to refer for psychiatric care.

\section{Measures}

\section{Exposure}

Our exposure of interest was the HAP intervention that was offered to participants in the experimental arm of the trial only.

\section{Outcome}

For our analysis, our primary outcome was the PHQ-9 score at 12 months. Response options generate a continuous score ranging from 0-27 since each of the nine items can be scored from zero (no symptoms) to three (nearly every day). Scores between ten and 14 represent moderate depression, and scores between 15 and 27 moderately severe to severe depression symptoms.(21)

\section{Mediators}

Causal mediation analyses require obtaining estimates in both the exposed participants, as well as the unexposed (i.e. the counterfactual). Where mediators were measured in both the experimental and control arms, estimates from participants in the control arm can be treated as the unexposed. However, where a mediator was only measured in the experimental arm, it was necessary to create a separate category for participants who had not been exposed to the mediator of interest but were still in the experimental arm.

\section{Therapeutic process indicators (M1)}


Characteristics associated with the delivery of the sessions were measured for participants in the experimental arm only. The first characteristic that we accounted for is the number of sessions completed that are categorised to reflect the phases of the HAP intervention that a participant completed (M1a: no sessions (unexposed); sessions one and two (phase 1), sessions three and four (phase 2); sessions five through eight (phase 3).

The second characteristic of the sessions that we accounted for is a participant's self-reported completion of assigned tasks outside of sessions (homework) to improve activity levels. At each session except for the first one, activity monitoring charts were completed indicating whether a participant completed homework outside of the sessions. These self-reported activity charts were scored using the following criteria: completely (scored 2), partially (scored 1), or not at all (scored 0 ). Based on this variable, we calculated a score representing the proportion of homework completed (M1b: $0=$ none (unexposed); $1=>0 \%$ and $\leq 50 \% ; 2=>50 \%$ ).

\section{Level of behavioural activation (M2)}

Behavioural activation was measured for participants in both the experimental (exposed) and control arms (unexposed) at three months after the trial started. An adapted version of the Behavioural Activation for Depression Scale Short Form (BADS-SF) was used to capture activity levels that reflect the level of behavioural activation due to the HAP intervention.(22)

\section{Extra sessions received in instances of non-response to the intervention (M3)}

Adding extra sessions for participants who do not respond to the intervention may help to improve symptoms of depression. Therefore, if a participant did not respond to the intervention by session five, they were offered two additional sessions. Estimating this indirect effect via the additional sessions involves two variables including non-response to treatment (M3a: 0=non-response (unexposed); $1=$ responded to treatment) and the number of extra sessions received (M3b: $0=$ no extra sessions (unexposed); 1 =one extra session; $2=$ two extra sessions). Appendix 1 details how non-response to the sessions was determined.

\section{Mediator-outcome confounders}

Due to the randomised nature of the exposure, it was not necessary to account for confounders for the association between the exposure and the outcome, or between the exposure and the mediators. However, it was necessary to account for confounders potentially distorting the association between the mediator and the outcome (mediator-outcome confounders). We considered all demographic characteristics as potential mediator-outcome confounders. The selection process for these confounders is described in the section on statistical methods.

\section{Statistical methods}




\section{General}

To better understand the relationship between different mediators and depression outcomes, we compared characteristics of the sessions (i.e. M1a - number of sessions and M1b proportion of homework completed), behavioural activation (M2), non-response to the intervention (M3a), and the number of extra sessions attended for participants who did not respond to HAP (M3b), with the outcome remission from depression (determined by a PHQ-9 score less than 10) for participants in the experimental arm only. Differences in baseline characteristics between treatment arms can be found in previous publications. $(12,16)$

\section{Mediation analysis}

We aimed to investigate the extent to which symptoms of depression measured at 12 months using the PHQ-9 questionnaire, were explained by the direct effect and indirect effects of the intervention (Figure 1). To achieve these objectives, we used the interventional (in)direct effects approach to mediation analysis to understand population level effects relevant to this analysis.(17) Findings for this analyses are reported according to guidelines for reporting mediation analyses (AGReMA statement).(23)

\section{Decomposition of total effect of the HAP intervention into direct and indirect effects}

The first step of the mediation analyses involved decomposing the total effect of the HAP intervention, into path-specific indirect effects and the direct effect. In order for the decomposition to be valid, the sum of the different path specific effects including the direct effect, through which the effects of the intervention is mediated, must be the same as the total effect of the intervention.(24)

With three mediators, the following decompositions are required to sum the total effect: an estimate that does not involve any mediators (level 0 - all mediators set to unexposed); an estimate that includes one mediator (level 1 - one mediator exposed, the rest unexposed); two mediators (level 2 - two mediators set to exposed, one to unexposed), and three mediators (level 3 - all three mediators set to exposed).

Setting a mediator at an exposed status indicates the pathway includes the effect of this mediator. Likewise, setting a mediator to an unexposed status, indicates the pathway excludes the effect of this mediator (i.e. setting M2 and M3 to unexposed indicted the pathway includes M1 only). Table 1 describes the total effect and decompositions for the direct and indirect effects of the HAP intervention that sum the total effect. 
Table 1

Decompositions for the direct and indirect effects of the HAP intervention that sum the total effect

\begin{tabular}{|c|c|c|c|c|}
\hline Estimate & Description & M1 & M2 & M3 \\
\hline Total effect & $\begin{array}{l}\text { Difference between predicted PHQ-9 } \\
\text { scores between the experimental and } \\
\text { control arm }\end{array}$ & NA & NA & NA \\
\hline Direct effect & $\begin{array}{l}\text { Difference between predicted PHQ-9 } \\
\text { scores in the intervention and control } \\
\text { arms, whilst fixing mediators to } \\
\text { unexposed status (level 0) }\end{array}$ & unexposed & unexposed & unexposed \\
\hline $\begin{array}{l}\text { M1 - } \\
\text { characteristics of } \\
\text { the sessions }\end{array}$ & $\begin{array}{l}\text { Difference between predicted PHQ-9 } \\
\text { scores when changing M1 from } \\
\text { levels in the exposed, to levels in the } \\
\text { unexposed, in the experimental arm } \\
\text { (level one) }\end{array}$ & $\begin{array}{l}\text { exposed to } \\
\text { unexposed }\end{array}$ & unexposed & unexposed \\
\hline $\begin{array}{l}\mathrm{M} 2 \text { - levels of } \\
\text { behavioural } \\
\text { activation }\end{array}$ & $\begin{array}{l}\text { Difference between predicted PHQ-9 } \\
\text { scores when changing M2 from } \\
\text { levels in the exposed, to levels in the } \\
\text { unexposed, in the experimental arm } \\
\text { (level 2) }\end{array}$ & exposed & $\begin{array}{l}\text { exposed to } \\
\text { unexposed }\end{array}$ & unexposed \\
\hline $\begin{array}{l}\text { M3 - additional } \\
\text { sessions offered } \\
\text { in instances of } \\
\text { non-response }\end{array}$ & $\begin{array}{l}\text { Difference between predicted PHQ-9 } \\
\text { scores when changing M3 from } \\
\text { levels in the unexposed, to levels in } \\
\text { the exposed, in the experimental arm } \\
\text { (level 3) }\end{array}$ & exposed & exposed & $\begin{array}{l}\text { exposed to } \\
\text { unexposed }\end{array}$ \\
\hline
\end{tabular}

\section{Estimation}

Estimation for the different effects was based on Monte Carlo integration using 1,000 fold expanded dataset. Estimates for the total, direct, and indirect effects were obtained by running regression models for the outcome of PHQ-9 score, separately in the exposed and unexposed, whilst setting the mediators at a random subject-specific distribution (Table 1). Further details of the estimation methods can be found in Appendix 1.

\section{Model fit}

Regression models used to estimate the total, direct and indirect effects, included any mediator-outcome confounder that improved model fit as indicated by the Akaike Information Criterion (AIC).(25) Results indicate that age, education, and baseline PHQ-9 scores are important confounders. These models also included mediators set to relevant exposed/unexposed status (Table 1).

The regression models used to set the mediators at random, subject-specific draws for the unexposed/exposed status, included any variable that improved model fit as indicated by the Akaike Information Criterion (AIC),(25) but not known to be potentially influenced by them. This ensured that mediator values drawn were more specific to the considered individual, thereby providing better insight 
into mechanism. Models for the different mediators used a combination of predictors including age, education, baseline PHQ-9 scores, participants expectations of treatment, and marital status. Any relevant non-linearities and interactions were included in all models if determined to be significant at the five percent level, using the post-estimation testparm command in Stata.

\section{Assumptions}

The interventional effects have important underlying assumptions that will influence the validity of our findings if violated. Reassuringly, due to the randomised nature of the HAP trial, many of the assumptions with the interventional effects are fulfilled. The interventional effects capture the components of the total effect mediated by the different mediators, even when the structural dependence between multiple mediators is unknown (i.e. direction of the causal effects between the multiple mediators is unknown, or if there is unmeasured common causes of the mediators (Figure 1)). The main assumption relevant to our study is that there are no unmeasured mediator-outcome confounders.

\section{Missing data}

There were missing data for the BADS-SF variable (measuring M2) at three months $(n=28,5.7 \%)$ and the PHQ-9 variable at 12 months ( $n=47,9.3 \%)$. To account for this, we implemented single stochastic imputation using chained equations with 10 burn-in iterations, under the assumption that data was missing at random (MAR). Details of the missing data analysis can be found in Appendix 2.

\section{Ethical approval and consent}

The trial protocol received ethical approval from the Sangath and LSHTM Institutional Review Boards. (18) Written or witnessed verbal (if the participant is illiterate) informed consent was mandatory for enrolment. All consent procedures were audio-taped, with the patient's approval, for quality assurance.

\section{Patient public involvement}

As this is a secondary data analysis of the original trial, patients and public were not involved

\section{Results}

There were 493 participants included in the study, with 248 (50\%) allocated to the control arm with EUC alone, and 245 (50\%) to the experimental arm with EUC plus HAP. Table 2 compares mediators measured at three months, between participants with remission from depression (PHQ-9<10) and participants with depression (PHQ-9 >=10) measured at 12 months after the trial started, in the experimental arm only. Results suggest participants who completed more homework and attended more sessions (M1), were less likely to recover from depression. Findings suggest at 12 months, participants who had sustained remission from depression, had higher mean behavioural activation levels measured at three months (compared to participants who did not recover from depression (M2). 
Page 10/19 
Table 2

Comparison of mediators between participants with and without remission from depression at 12 months, in the experimental arm of the trial only

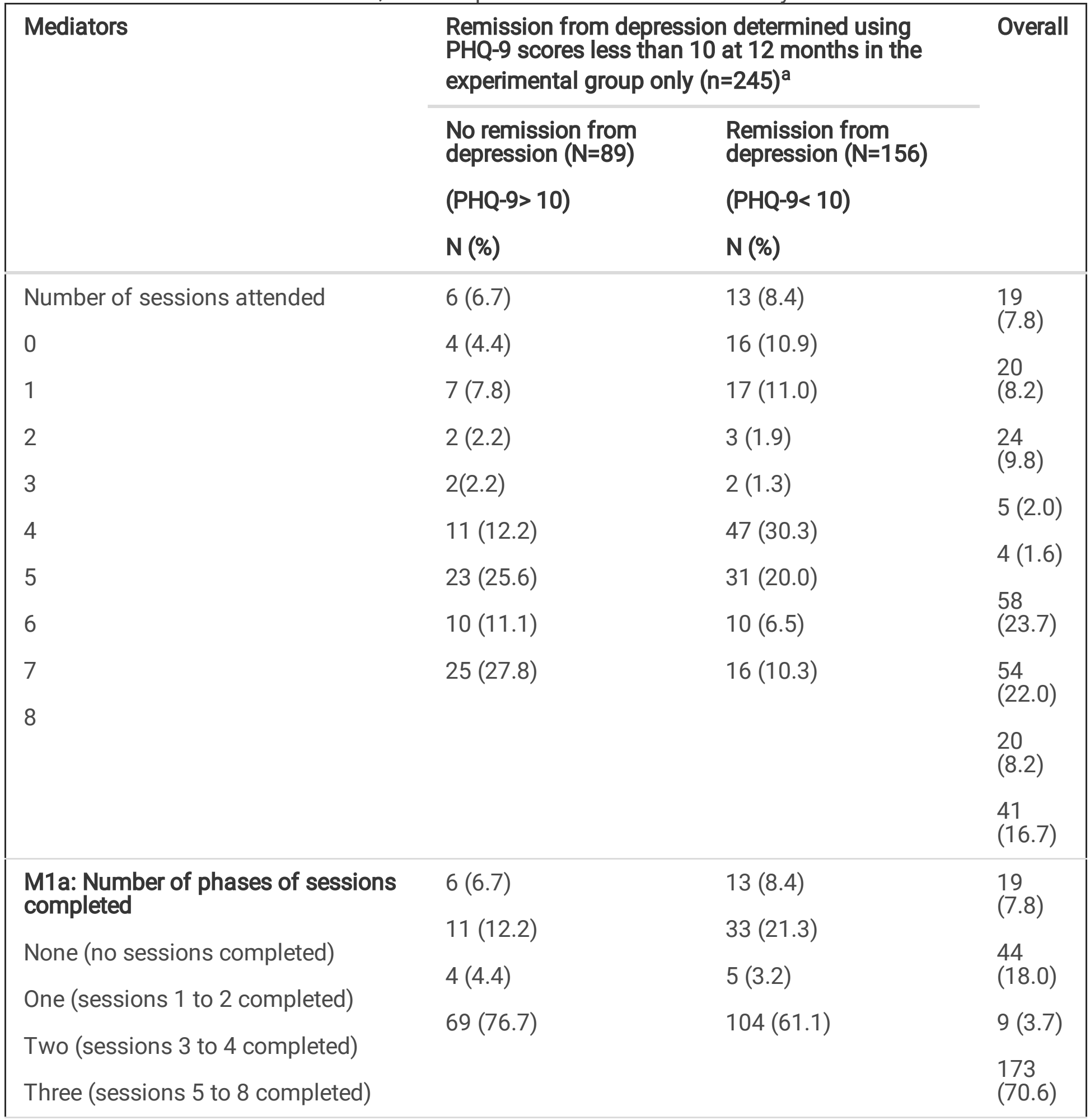

a Missing data has been imputed by trial arm, using single imputation stochastic models adjusted for any factors that could potentially influence missingness

b Table 1 describes how non-response to counselling was determined 


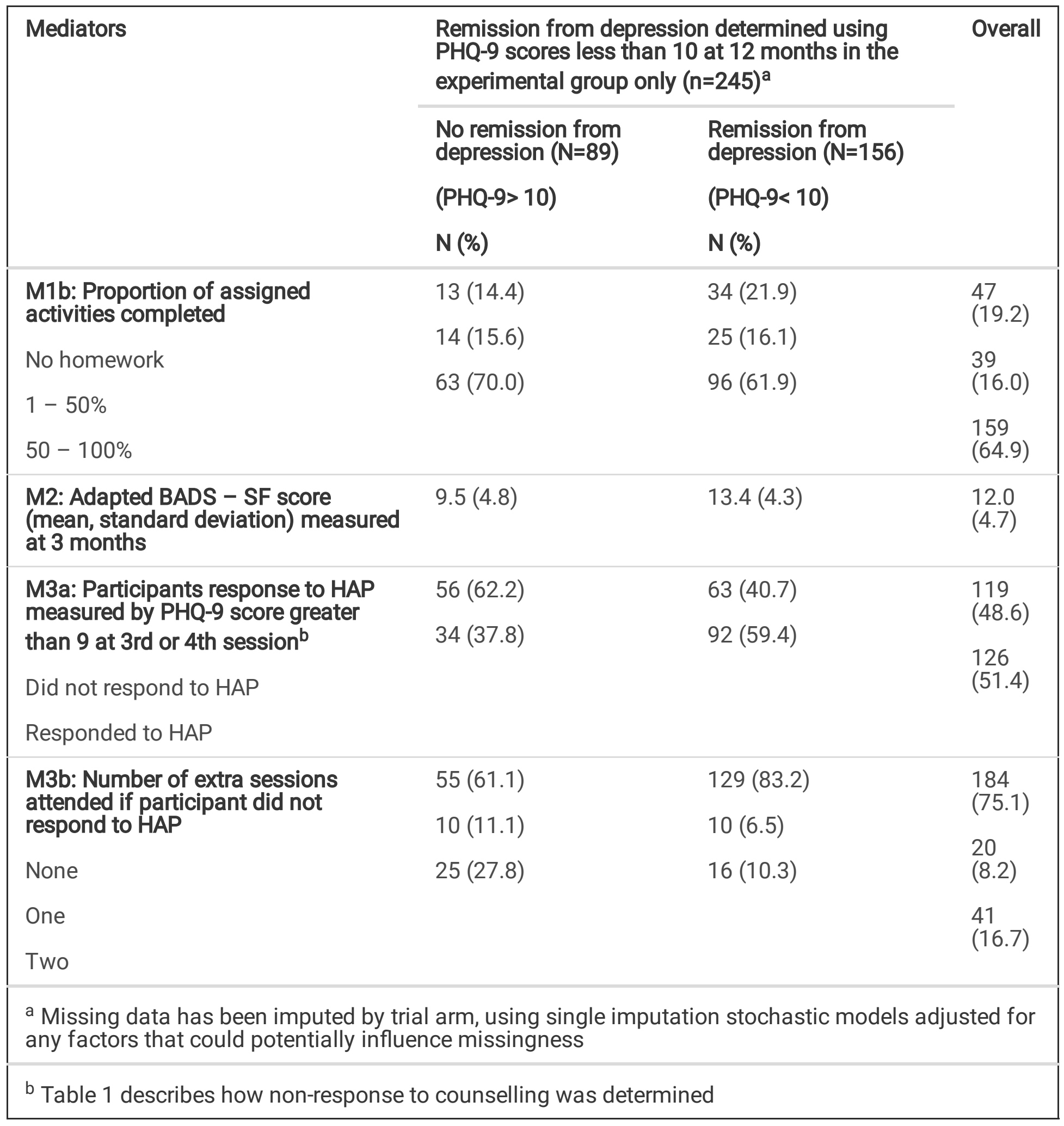

\section{Mediation}

Table three demonstrates that at 12 months, the total mean difference in PHQ-9 scores between the experimental and control arms (adjusted mean difference in PHQ-9 scores: -2.2 , bias corrected $95 \% \mathrm{Cl}$ : $-3.3,-0.8), 45 \%$ was mediated through indirect effects via activity levels measured using the adapted version of the BADS-SF (adjusted mean difference in PHQ-9 scores attributable to pathways via BADS-SF 
$-1.0,-1.3,-0.6)$. There was no evidence to support mediation through indirect effects via characteristics of the sessions including number of sessions and proportion of homework completed $(2.9,-1.3,5.0)$. The additional sessions offered to non-responders to the intervention did not improve depression scores either $(-0.8,-2.1,1.2)$. Findings also suggest that the HAP intervention improves symptoms of depression through mechanisms other than those explained with our mediators $(-3.3,-5.6,-0.5)$.

Table 3

Total effect and interventional in(direct) effects for the HAP intervention at 12 months

\begin{tabular}{|c|c|}
\hline Effect & $\begin{array}{l}\text { Estimates (bias-corrected } 95 \% \\
\mathrm{Cl} \text { ) } \mathrm{a}, \mathrm{b}, \mathrm{c}, \mathrm{d}\end{array}$ \\
\hline Total effect & $-2.2(-3.3$ to -0.8$)$ \\
\hline Interventional direct effect & $-3.3(-5.6$ to -0.5$)$ \\
\hline Interventional indirect effect through sessions only (M1) & $2.9(-1.3$ to 5.0$)$ \\
\hline Interventional indirect effect through BA levels only (M2) & $-1.0(-1.3$ to -0.6$)$ \\
\hline Interventional indirect effect through extra sessions & $-0.8(-2.1$ to 1.2$)$ \\
\hline $\begin{array}{l}\text { Interventional indirect effect through the dependence of BA levels } \\
\text { on sessions (M3) }\end{array}$ & $0.0(-0.6$ to 0.7$)$ \\
\hline
\end{tabular}

${ }^{\text {a }}$ Estimates have been adjusted for mediator-outcome confounders of baseline PHQ-9 scores, age and education.

${ }^{b}$ Estimation for the different effects was based on Monte Carlo integration using 1,000 fold expanded dataset

${ }^{c}$ Bias-corrected confidence intervals were based on nonparametric bootstrap with 1000 resamples

d Missing data has been imputed by trial arm, using single imputation stochastic models adjusted for any factors that could potentially influence missingness

\section{Discussion}

Applying robust, interventional (in)direct effects has allowed us to understand what components of the HAP intervention are effective, and what other components may need to be modified before considering scale-up. Specifically, the indirect effect mediated through behavioural activation improved symptoms of depression. However, the indirect effect mediated through both the characteristics of the sessions and the extra sessions offered to participants who did not respond to the intervention, did not appear to improve symptoms of depression. 
The main strategy included in the HAP intervention was behavioural activation for depression.(19) The results from our analysis support this approach, whereby levels of behavioural activation captured using the adapted BADS-SF, improved symptoms of depression. Our findings were also supported by a Cochrane systematic review that found behavioural activation may be more effective than medication in improving symptoms of depression.(26) However, results from other mediation analyses evaluating the role of behavioural activation are mixed.(27) Nevertheless, comparisons are difficult given the different contexts, population under investigation, study designs, conditions being treated, interventions provided, and methods used to conduct the analyses.

Our estimates suggest that characteristics of the sessions we had available, including the number of phases of the intervention the participant attended and the proportion of homework completed, did not influence depression outcomes. Results from the univariate analysis can help to explain this issue where participants who attended more sessions and completed more homework, were less likely to recover from depression. Likewise, participants were less likely to recover from depression, if they completed more than $50 \%$ of their homework, compared to participants who completed less homework. A likely explanation for this phenomenon is that participants who received a more intense intervention had more severe forms of depression and might have needed to continue therapy because of non-response. To address this matter, we created an additional mediator to estimate the role of the extra sessions attended by participants who did not respond to the intervention, in improving symptoms of depression. Our findings did not indicate any benefit to receiving the extra sessions suggesting that if the HAP intervention is brought to scale, it will be important to bear this in mind when deciding on how to improve symptoms of depression among non-responders.

Different theoretical approaches to implementation research, other than those driven by a positivist, deductive approach may help to address these findings. As an example, a realist evaluation can be used to better understand why some participants did not respond to the intervention, and under what circumstances.(28) Implementation science theories and frameworks can also be used to help inform why the extra sessions did not improve symptoms of depression.(29) Findings from such investigations could then be used to modify the intervention and future trials explore the effectiveness of the adaptations.

Estimates for our direct effect, indicates that a large proportion of the total effect is still unknown. These results are key, as they suggest that there were characteristics of the HAP intervention not captured with the mediators that we had available, that helped to improve symptoms of depression. Indeed, there were four domains of strategies included as part of the sessions: engagement (psychoeducation, family psychoeducation and treatment planning); behavioural activation; need-based strategies (i.e. addressing interpersonal triggers, problem-solving, relaxation), and social integration.(19) It is entirely conceivable that the effect of the domains not captured with the mediators we had available, was expressed through the direct effect. This issue highlights the importance of identifying and subsequently collecting relevant data on potential mediators when planning studies. This could provide greater insights into how the intervention can be optimised for future scale-up.

Page 14/19 


\section{Limitations}

When applying the interventional effects to a randomised trial, the main underlying assumption is that all important mediator-outcome confounders are accounted for. Failing to do so, can potentially bias all estimates including the direct and indirect effects. As an example, non-compliers are often quite different from compliers in terms of depression that may distort the relationships between the direct and indirect effects.

Findings of our mediation analyses need to be interpreted with caution when generalising to different contexts. However, by conducting robust mediation analyses for similar interventions in different contexts, we will be able to better understand what components of the intervention worked for whom and under what circumstances.

\section{Conclusions}

This paper uses a robust approach to mediation analysis to understand how complex psychological therapies work and under what circumstances, something that can potentially be used to inform policy. $(17,30)$ Not only do our findings reinforce the role of behavioural activation found with other mediation analyses, $(16,31)$ but we now understand in the context of the HAP trial, that the number of sessions attended and the amount of homework completed is not necessary indicative of an improvement in symptoms of depression symptoms. Moreover, estimates suggest that attending an additional one to two sessions if participants do not respond to the intervention did not improve symptoms of depression. More is needed to understand how interventions such as HAP, can be adapted, in order to improve outcomes in the non-responders.

\section{Abbreviations}

Low- and middle-income countries

LIMCs

World Health Organization

WHO

WHO's Mental Health Gap Action Programme

mhGAP

The Healthy Activity Programme

HAP

Behavioural Activation for Depression Score

BADS-SF

Patient Health Questionnaire 9

PHQ-9

\section{Declarations}


Ethics approval: The trial protocol received ethical approval from the Sangath and LSHTM Institutional Review Boards.(18)

Consent for publication: not applicable

Data availability: Data is owned by a third part. Data will be shared on request to the corresponding author with person of the third part. Code is available upon request.

Competing interests: None declared

Funding: This work was supported by: Nadine Seward supported by the National Institute for Health Research (NIHR) Global Health Research Unit on Health System Strengthening in Sub-Saharan Africa, King's College London (GHRU 16/136/54). Darío Moreno-Agostino is supported by the ESRC Centre for Society and Mental Health at King's College London (ESRC Reference: ES/S012567/1). The views expressed are those of the author(s) and not necessarily those of the ESRC or King's College London.

\section{Author contributions:}

Conceptualised the idea for the study: NS, RA

Wrote initial and subsequent drafts of the paper: NS

Statistical guidance: SV

Read and edited all drafts of the paper: NS, RA, SV, DM

Provided guidance on the original trial and read/edited subsequent drafts of the paper: VP

Acknowledgments: We would like to acknowledge the participants in the original trial as well as authors who wrote the protocol paper and subsequent papers for the intervention development and trial findings. We would also like to acknowledge the Wellcome Trust Senior Research Fellowship grant to VP (091834) to carry out the original work.

\section{References}

1. Patel V, Chisholm D, Parikh R, et al. Addressing the burden of mental, neurological, and substance use disorders: key messages from Disease Control Priorities, 3rd edition. Lancet (London, England). 2016;387(10028):1672-85.

2. World Health Organization. MhGAP. Mental Health Gap Action Programme. Scaling up care for mental, neurological and substance use disorders. Geneva; 2008.

3. Patel V. The need for treatment evidence for common mental disorders in developing countries. Psychological medicine. 2000;30(4):743-6. 
4. Van Ginneken N, Tharyan P, Lewin S, et al. Non-specialist health worker interventions for the care of mental, neurological and substance-abuse disorders in low-and middle-income countries. Cochrane database of systematic reviews. 2013(11).

5. Singla D, Kohrt B, Murray L, et al. Psychological Treatments for the World: Lessons from Low- and Middle-Income Countries. Ann Rev Clin Psychol. 2017;13:149-81.

6. Saxena S, Thornicroft G, Knapp M, et al. Resources for mental health: scarcity, inequity, and inefficiency. Lancet. 2007;370(9590):878-89.

7. Patel V, Saxena S, Lund C, et al. The Lancet Commission on global mental health and sustainable development. The Lancet. 2018;392(10157):1553-98.

8. Arjadi R, Nauta MH, Scholte WF, et al. Internet-based behavioural activation with lay counsellor support versus online minimal psychoeducation without support for treatment of depression: a randomised controlled trial in Indonesia. Lancet Psychiatry. 2018;5(9):707-16.

9. Chibanda D, Weiss H, Verhey R, et al. Effect of a Primary Care-Based Psychological Intervention on Symptoms of Common Mental Disorders in Zimbabwe: A Randomized Clinical Trial. JAMA. 2016;316(24):2618-26.

10. Gureje 0 , Oladeji BD, Montgomery AA, et al. High-versus low-intensity interventions for perinatal depression delivered by non-specialist primary maternal care providers in Nigeria: cluster randomised controlled trial (the EXPONATE trial). Br J Psychiatry. 2019;215(3):528-35.

11. Behavioral activation treatment for depression. Returning to contextual roots [press release]. United Kingdom: Blackwell Publishing; 2001.

12. Patel V, Weobong B, Weiss $H$, et al. The Healthy Activity Program (HAP), a lay counsellor-delivered brief psychological treatment for severe depression, in primary care in India: a randomised controlled trial. The Lancet. 2017;389(10065):176-85.

13. Sikander S, Ahmad I, Atif N, et al. Delivering the Thinking Healthy Programme for perinatal depression through volunteer peers: a cluster randomised controlled trial in Pakistan. The Lancet Psychiatry. 2019;6(2):128-39.

14. Araya R, Menezes PR, Claro HC, et al. Effect of a Digital Intervention on Depressive Symptoms in Patients With Comorbid Hypertension or Diabetes in Brazil and Peru: Two Randomized Clinical Trials. JAMA. 2021;325(18):1852-62.

15. Dunn G, Fowler D. R R, et al. Effective elements of cognitive behaviour therapy for psychosis: results of a novel type of subgroup analysis based on principal stratification. Psychological medicine. 2012;42(5):1057-68.

16. Weobong B, Weiss $H, M c D a i d ~ D$, et al. Sustained effectiveness and cost-effectiveness of the Healthy Activity Programme, a brief psychological treatment for depression delivered by lay counsellors in primary care: 12-month follow-up of a randomised controlled trial. PLOS Medicine. 2017;14(9):e1002385.

17. Vansteelandt S, Daniel RM. Interventional effects for mediation analysis with multiple mediators. Epidemiology (Cambridge Mass). 2017;28(2):258-65. 
18. Patel V, Weobong B, Nadkarni A, et al. The effectiveness and cost-effectiveness of lay counsellordelivered psychological treatments for harmful and dependent drinking and moderate to severe depression in primary care in India: PREMIUM study protocol for randomized controlled trials. Trials. 2014;15:101.

19. Chowdhary N, Anand A, Dimidjian S, et al. The Healthy Activity Program lay counsellor delivered treatment for severe depression in India: systematic development and randomised evaluation. $\mathrm{Br} \mathrm{J}$ Psychiatry. 2016;208(4):381-8.

20. Singla D, Weobong B, Nadkarni A, et al. Improving the scalability of psychological treatments in developing countries: an evaluation of peer-led therapy quality assessment in Goa, India. Behav Res Ther. 2014;60(100):53-9.

21. Kroenke K, Spitzer R, Williams J. The PHQ-9: validity of a brief depression severity measure. J Gen Intern Med. 2001;16(9):606-13.

22. Manos R, Kanter J, Luo W. The behavioral activation for depression scale-short form: development and validation. Behavior therapy. 2011;42(4):726-39.

23. Lee H, Cashin A, Lamb S, et al. A Guideline for Reporting Mediation Analyses of Randomized Trials and Observational Studies: The AGReMA Statement. JAMA. 2021;326(11):1045-56.

24. Daniel R, De Stavola B, Cousens $S$, et al. Causal mediation analysis with multiple mediators. Biometrics. 2015;71(1):1-14.

25. Burnham K, Anderson D. Multimodel Inference: Understanding AIC and BIC in Model Selection. Sociological Methods Research. 2004;33(2):261-304.

26. Uphoff E, Ekers D, Robertson L, et al. Behavioural activation therapy for depression in adults. Cochrane Database of Systematic Reviews. 2020(7).

27. Janssen NP, Hendriks GJ, Baranelli CT, et al. How Does Behavioural Activation Work? A Systematic Review of the Evidence on Potential Mediators. Psychother Psychosom. 2021;90(2):85-93.

28. Pawson R, Tilley N. Realistic evaluation. London: Sage; 1997.

29. Nilsen P. Making sense of implementation theories, models and frameworks. Implementation Science. 2015;10(1):53.

30. VanderWeele T. Policy-relevant proportions for direct effects. Epidemiology (Cambridge Mass). 2013;24(1):175-6.

31. Singla D, MacKinnon D, Fuhr D, et al. Multiple mediation analysis of the peer-delivered Thinking Healthy Programme for perinatal depression: findings from two parallel, randomised controlled trials. The British Journal of Psychiatry. 2021;218(3):143-50.

\section{Figures}




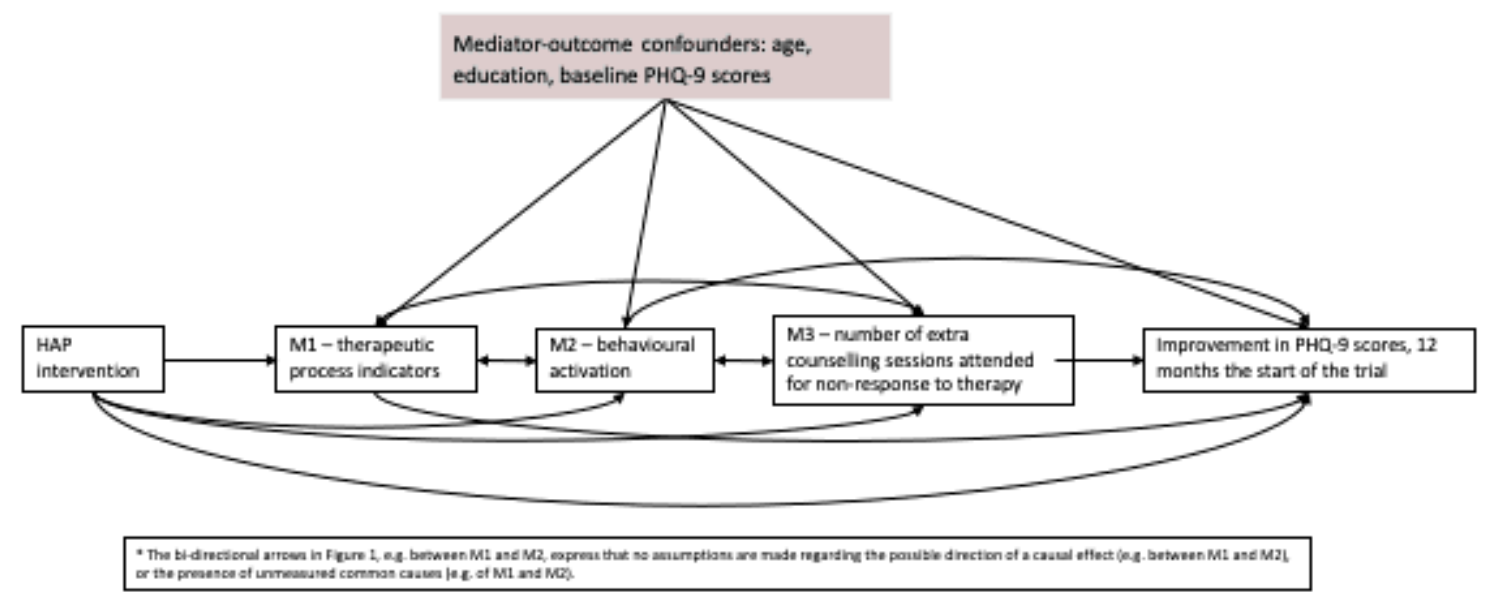

Figure 1

The main assumption relevant to our study is that there are no unmeasured mediator-outcome confounders.

\section{Supplementary Files}

This is a list of supplementary files associated with this preprint. Click to download.

- Appendix1.docx

- Appendix2.docx 